\title{
Developmental instability as an estimator of genetic stress
}

\author{
C Pertoldi ${ }^{1,2}$, TN Kristensen ${ }^{1,3}$, DH Andersen ${ }^{4}$ and V Loeschcke ${ }^{1}$ \\ ${ }^{1}$ Department of Ecology and Genetics, University of Aarhus, Ny Munkegade, Building 540, DK-8000 Aarhus C, Denmark; ${ }^{2}$ Department \\ of Wildlife Ecology and Biodiversity, National Environmental Research Institute, Kalø Grenåvej 14, DK-8410 Rønde, Denmark; \\ ${ }^{3}$ Department of Genetics and Biotechnology, Danish Institute of Agricultural Sciences, P.O. Box 50, DK-8830 Tjele, Denmark; \\ ${ }^{4}$ Dipartimento di Biologia Evoluzionistica Sperimentale, Via Selmi 3, I-40126 Bologna, Italy
}

To set conservation priorities, scientists should be able to assess the relative threats posed by the effects of loss of genetic variability, inbreeding and outbreeding as these can generate 'genetic stress'. Developmental instability (DI) has been suggested as an indicator of stress, possibly being more sensitive than other measures. However, there is controversy as to whether DI is an accurate and reliable tool for assessing the degree of genetic stress. After 50 years of the presentation of
Lerner's conjecture, there are still several unresolved questions about the relationship between $\mathrm{DI}$ and genetic stress. Here, we review studies on mechanisms behind DI. The current status on the use of DI as an indicator of genetic stress is discussed, and suggestions are presented on how to obtain more knowledge on the potential of $\mathrm{DI}$ in an evolutionary context.

Heredity (2006) 96, 122-127. doi:10.1038/sj.hdy.6800777; published online 7 December 2005

Keywords: Lerner's conjecture; homeostasis; admixture analysis; heterozygosity; environmental variability

\section{Introduction}

Genetic stress and its consequences on fitness The effects of human activity on natural ecosystems have increased the risk of extinction for many animal and plant populations. Human activities have caused an increase in stochastic fluctuations in population sizes, changed the interactions between predators and their prey and led to changes in the genetic structure within and between populations. These factors could potentially result in inbreeding and/or outbreeding depression and loss of genetic variation (Crnokrak and Roff, 1999; Frankham, 2005). For these and other reasons, a fitness decline has accelerated during the last decades in many populations and this process is likely to continue in the future. To set conservation priorities, it is important to assess the relative threats posed by the different factors causing fitness declines. Conservation biologists need rapid and reliable techniques for detecting reductions in fitness inflicted by demographic and environmental insults. From a genetic point of view, acquiring more knowledge about how to detect inbreeding and outbreeding depression and loss of genetic variability under natural conditions at an early stage and to evaluate its consequences are the main priorities. Recognising the presence of genetic and environmental stresses before their effects become deleterious is one of the most important but at the same time difficult tasks (Gilligan et al, 2000; Lens et al, 2002a).

Correspondence: C Pertoldi, Department of Ecology and Genetics, University of Aarhus, Ny Munkegade, Building 540, DK-8000 Aarhus C, Denmark.E-mail: biocp@nf.au.dk

Received 30 August 2005; accepted 26 October 2005; published online 7 December 2005
Developmental instability as an indicator of stress Developmental instability (DI) refers to an individual's inability to produce a specific phenotype under a given set of environmental conditions. A number of studies have shown that DI is positively associated with the level of stress that individuals experience (Palmer, 1994; Lens et al, 2002a). Therefore, DI has been suggested as a sensitive method for use in population surveys that would be valuable in indicating whether individuals or populations are harmed by the presence of (genetic) stress (Gilligan et al, 2000; Lens et al, 2002a, b). However, to validate the use of DI as a decision tool in conservation biology, we need to know its accuracy and reliability as an indicator of stress.

The genetic basis of increased DI in genetically stressed organisms

Developmental and evolutionary biologists have debated the genetic basis of DI for more than 50 years. An increase in DI as a consequence of genetic stress has been explained by three hypotheses: (a) the genomic coadaptation theory, which predicts that more balanced coadapted gene complexes, established over the evolutionary history of an organism via natural selection, will show higher stability in development over time (reviewed in Markow, 1995); (b) the heterozygosity theory, predicting that levels of heterozygosity will be inversely correlated with levels of DI (Lerner, 1954; Livshits and Kobyliansky, 1985), as heterozygosity has been suggested to have a buffering role through increased biochemical diversity and thus enabling a dynamic stable developmental pathway in changing environments (Livshits and Smouse, 1993); and (c) 
Rutherford and Lindquist (1998) presented evidence that Hsp90 acts as a capacitor of evolutionary change, and Rutherford (2003) suggested that under conditions where cellular levels of Hsp90 become limited (which may be the case when organisms are exposed to genetic stress), an increase in DI will be observed.

A controversial issue, which contributes to the confusion concerning the relationship between DI and genetic stress, is the ongoing discussion dealing with the overdominance hypothesis vs the partial dominance hypothesis in explaining inbreeding depression. The first theory suggests that the capability to buffer biochemical pathways against negative genetic effects during ontogenesis is caused by a diversity of biochemical products resulting from heterozygous genotypes at unlinked loci. The latter theory (partial dominance) explains heterozygote advantage with an increased expression of recessive deleterious alleles with increased homozygosity. When expressed, such rare deleterious alleles would be detrimental to metabolic processes (Roff, 1998).

According to the overdominance hypothesis, fitness (biochemical efficiency) will always decrease with an increase in homozygosity. In consequence, DI would be expected to increase. However, with partial dominance, a decrease in fitness with decreased heterozygosity will not necessarily be observed because the deleterious alleles can be purged from the population. Therefore, with partial dominance, the association between DI and homozygosity may be very complex.

Both the overdominance and the partial dominance hypotheses, however, predict enhanced growth and reduced DI at high levels of heterozygosity. The heterozygous individuals would then typically be larger, which would affect both the common estimators of DI: phenotypic variability $\left(\sigma^{2} p\right)$ and fluctuating asymmetry (FA) (Pertoldi et al, 2003). Fluctuating asymmetry is a controversial topic. Some research, particularly in the fields of sexual selection and conservation, has suggested that the levels of stress and FA are positively correlated (eg Parsons, 1992, but see others, eg Clarke, 2003; Kruuk et al, 2003; Leung et al, 2003).

\section{Genetic factors and extinction}

Lowered fitness in small isolated populations is often suggested as an important factor in the final extinction of an endangered species (Hedrick and Miller, 1992; Spielman et al, 2004; Frankham, 2005). In addition, progeny from matings between populations that are genetically differentiated may reduce fitness. These effects may be a significant problem in designing strategies for the reintroduction and repopulation of endangered species (Lynch, 1991). The overall effects of inbreeding or outbreeding on fitness may vary between different components of fitness. There may also be ascertainment bias, for example, the impact of inbreeding on survivorship is generally measured, but other components of fitness such as fecundity and mating success may also be affected but are more difficult to detect (eg Miller and Hedrick, 1993). However, Falconer and Mackay (1996) argue that inbreeding will on average lead to a decrease in fitness for all traits affected by dominance. Even though there are controversies about the importance of inbreeding and outbreeding depression within the field of conservation biology, there are no doubts that their effects on endangered species is fundamental to determining populations' potential for long-term persistence. There is also no doubt that if DI could be considered a reliable indicator of genetic stress, it could serve as a useful index to identify populations that are, or soon will become, subject to inbreeding or outbreeding depression. As such, it could potentially be a significant instrument to monitor the well being of small endangered populations (Lens et al, 2000).

\section{Fluctuating asymmetry as an estimator of DI}

One common estimator of DI is FA. The estimation of FA has, however, several problems (Palmer, 1994), especially if the aim is to provide data on natural populations. One of the major problems is to distinguish between genetic and environmental sources that modify the FA level (Kristensen et al, 2004). In laboratory populations, the genetic component of FA can be inferred by manipulating levels of inbreeding and outbreeding within and between populations by means of controlled crosses. With this approach, it is also possible to minimize the macroenvironmental variance $\left(\mathrm{MACRO} \sigma^{2} e\right)$ between individuals by estimating FA under controlled greenhouse or laboratory environments. However, distributions that depart from the normal distribution with mean equal to 0 can provide unreliable estimates of levels of DI if directional asymmetry or antisymmetry has a genetic basis (Palmer, 1996).

\section{Empirical findings on the association between $\mathrm{DI}$ and the level of genetic stress}

Despite several methodological problems afflicting FA when used as a DI estimator, it has been used in several studies dealing with genetic stress. Many of these show that hybridization (outbreeding) or inbreeding effect FA in a highly unpredictable manner and that FA measurements can therefore not be considered a reliable indicator of disturbance in the genetic makeup (Lamb et al, 1990; Rao et al, 2002; Kruuk et al, 2003). However, when fitness traits themselves are investigated, inconsistencies in the effects of inbreeding and outbreeding are also found. This fact may reflect the complexity of the mechanisms involved in the relationship between genetic distance, genomic compatibility and hybrid fitness. Outbreeding depression is thought to result from several mechanisms including dilution of locally adapted genomes, underdominance and disruption of favourable epistatic interactions. The relationship between hybrid fitness and the genetic divergence between parental populations will thus depend on the mechanisms that generated this divergence (for a review, see Burke and Arnold, 2001). Interpopulation outcrossing often has positive effects on fitness-related traits. On the other hand, crosses between genetically differentiated populations can result in decreased fitness of the progeny and some studies have shown large fitness advantages of F1 hybrids over a wide range of genetic or geographic distances (Edmands, 1999). This heterosis effect is generally interpreted as the recovery from inbreeding depression caused by accumulation of deleterious alleles in isolated populations (Keller and Waller, 2002). In other studies, fitness was found to decline with increasing genetic distance between the parental populations, or fitness was highest 
at intermediate distances (for a review, see Waser and Williams, 2001).

\section{Methodological considerations on the use of FA and $\sigma^{2} p$ as estimators of DI}

Even if the explanations for inbreeding depression seem to be simpler (or at least better described) than those associated with outbreeding depression, the relationship between the level of inbreeding and/or loss of genetic variability and DI is also controversial (Clarke, 1998a, b). Several studies have indeed reported a positive relationship between DI and the level of inbreeding in a variety of animal species (eg Leary et al, 1983), whereas others do not (eg Gilligan et al, 2000; Taylor, 2001; Kruuk et al, 2003). The conflicting results are not surprising as many factors may confound the relationship between DI, inbreeding and fitness (Lens et al, 2002a,b). It has been suggested that the outcomes are taxon-, population-, trait- and environment specific (Vøllestad et al, 1999). Furthermore, interactions between genotype and environment might confound the relation between inbreeding and FA (Lens et al, 2002a), as it is well established that the degree of inbreeding depression expressed by inbred populations can depend on the environment (Keller and Waller, 2002).

Additional problems exist for the DI estimator $\sigma^{2} p$. This estimator could be considered as an ideal estimator of DI, as long as $\sigma^{2} p$ is estimated among genetically identical individuals $\left(\sigma^{2} g=0\right)$ reared under identical environmental conditions $\left(\sigma^{2} e \approx 0\right)$. Such conditions are of course very difficult to create (Pertoldi et al, 2001a, 2005a). In populations composed of genetically different individuals in a constant environment $\left(\sigma^{2} e \approx 0\right)$, the $\sigma^{2} p$ value of a population is roughly correlated with the $\sigma^{2} g$ (Pertoldi et al, 2003), assuming that the individual DIs are constant or negligible, as $\sigma^{2} p=\sigma^{2} g+\sigma^{2} e+\mathrm{G} \times \mathrm{E}+$ covGE + DI (Pertoldi et al, 2001a,b), where $\mathrm{G} \times \mathrm{E}$ is the genotype-environment interaction and covGE is the genotype-environment covariance. $\sigma^{2} p$ is influenced by $\sigma^{2} g$ and $\sigma^{2} e$ and inbreeding is also expected to affect the level of $\sigma^{2} p$ within a population, although predictions may vary depending on either the balance between the decrease in additive genetic variance $\left(\sigma^{2} a\right)$ through drift or changes in environmental variance associated with different levels of homozygosity (Whitlock and Fowler, 1999; Kristensen et al, 2005). FA may be a more reliable index than $\sigma^{2} p$, since the dissimilarity in expression of a given character on the left and the right side cannot be explained by either $\sigma^{2} g$ or $\sigma^{2} e$ as it is assumed that in bilateral traits, both sides of an individual are controlled by the same set of genes and are exposed to the same environmental conditions $\left(\sigma^{2} e=0\right)$ (Palmer, 1994).

However, because the estimation of individual DI by single trait asymmetry is an attempt to estimate $\sigma^{2} p$ with two data points (ie the left and right trait value), the correlation between individual asymmetry and the presumed underlying DI is weak. Extending the estimation of DI by means of FA to the population level further complicates the picture because of problems associated with among-individual heterogeneity $(\mathrm{EH})$ in the degree of DI. The EH can be due to the presence of $\sigma^{2} e$ within the population sample and/or to different susceptibility to stress of the genotypes within the population (Pertoldi et al, 2005a). Whitlock (1998) proposed a method, based on hypothetical repeatability of individual asymmetry to convert patterns in FA into patterns in the presumed underlying DI. The repeatability estimates the proportion of the total variation in the unsigned FA that results from between-individual heterogeneity in the underlying DI. A certain degree of heritability of FA is necessary if it is to be interpreted as a fitness indicator. From a theoretical basis, a non-zero heritability is problematic when FA is defined as a within-individual microenvironmental variance (Gavrilets and Hastings, 1994), indicating that it should only be used with caution to estimate DI.

The methodological difficulties mentioned above play important roles in explaining the inconsistency of the results on associations between DI and the level of inbreeding (or more general by genetic stress). The mean heterozygosity within a population is predicted to be negatively correlated with mean population FA and/or $\sigma^{2} p$. In investigations at the individual level, the proportion of heterozygous loci of an individual and its FA estimate is predicted to be negatively correlated. The main problem associated with these kinds of approaches is that the molecular markers used (mainly allozymes) to estimate heterozygosity may not be representative of the entire genome. Furthermore, there is the possibility that the markers are not selectively neutral (Vøllestad et al, 1999).

Tests of the relationship between heterozygosity and DI based on DNA variation may provide a more rigorous test of the hypothesis, and such investigations could shed light on possible differences between heterozygosities at coding and neutral loci. The latest meta-analysis conducted by Vøllestad et al (1999) on this issue showed a weak negative relationship between heterozygosity and DI. Despite this weak tendency, it is generally accepted that a negative relationship between heterozygosity and DI exists, but that it becomes apparent only in certain ecological and/or population contexts, possibly because it is concealed by various exogenic or endogenic factors.

\section{Experimental approaches and evidence for a relationship} between genetic variability and developmental instability Pertoldi et al (2003) presented the first study that actually applied a theoretical rational to the relationship between $\sigma^{2} g$ and DI, helping to understand why investigations dealing with the relationship between $\sigma^{2} g$ and DI have given controversial results. The simulation presented by Pertoldi et al (2003) showed that the hypothesized negative relationship between $\sigma^{2} g$ and DI can be counteracted by the observed positive relationship between $\sigma^{2} g$ and DI obtained from the simulation.

Experiments on cyclically parthenogenetic organisms, for example Daphnia or Apis mellifera, include analyses of differences in DI between males and females of haplodiploid taxa, or parthenogenetic and sexually reproducing individuals (Clarke, 1997; Crespi and Brett, 1997; Deng, 1997; Pertoldi et al, 2001b; Andersen et al, 2002). However, these studies have been limited to a few species and have been criticized because the parthenogenetic and the sexual populations are often allopatric. The comparison of DI among populations is therefore difficult, given that DI may be affected by environmental 
conditions that differ between the populations (see Pertoldi et al, 2001b).

A common observation is that progeny in the F1 generation after hybridization exhibit enhanced fitness and decreased levels of DI relative to their parents. This effect is generally believed to originate from increased heterozygosity (Ferguson et al, 1987). Two parental inbred lines which are crossed should give rise to an F1 generation that is developmentally more stable (smaller FA and smaller $\sigma^{2} p$ ) than the two parental lines, since it will be heterozygous at all the loci that are fixed for different alleles in the two parental lines. The main problem associated with these experiments is that often the parental lines are not totally inbred and therefore the residual variance, dominance variance $\left(\sigma^{2} D\right)$ and the interaction variance $\left(\sigma^{2} I\right)$, in the parental lines will confound the comparison of DI in the F1 generation. Indeed, to obtain inbred lines with an inbreeding level close to one, full sib matings have to be performed for at least 20 consecutive generations. During the process of inbreeding, there is potentially strong selection for heterozygotes at non-neutral loci, which means that the actual inbreeding coefficient might be lower than expected from standard formulae. However, the major problem with this approach is that even if $\sigma^{2} g=0$ in the two parental lines and consequently in the F1, there is no way of testing if the MACRO $\sigma^{2} e$ in the two parental lines was the same as in the F1 generation, which can obscure the hypothesized relationship between $\sigma^{2} g$ and DI.

\section{Discussion}

To obtain more knowledge on the association between genetic stress and DI, we suggest an expansion on the approach put forward by Pertoldi et al (2001a). The presence of $\mathrm{MACRO} \sigma^{2} e$ in a monoclonal population can be detected by calculating the covariance between right and left side of individuals $(\operatorname{cov}(r, l))$. This variation will blur the true association between DI and the level of genetic stress. When taking account of the $\mathrm{MACRO} \sigma^{2} e$, a clearer picture of the true association can be detected. This approach would be valid for monoclonal strains and for an F1 generation produced from two completely homozygous parental strains. If $\operatorname{cov}(r, l)$ is found in a monoclonal strain, it can only be due to macroenvironmental factors. If MACRO $\sigma^{2} e$ is present, it affects $\sigma^{2} p$ measures, as a positive cov $(r, l)$ will lead to an overestimation of $\sigma^{2} p$ and an underestimation of FA, whereas a negative covariance will underestimate $\sigma^{2} p$ and overestimate FA (Pertoldi et al, 2001a). Furthermore, $\mathrm{MACRO} \sigma^{2} e$ may often change the population trait mean. As the trait means are commonly interpreted as a change in the population fitness, it is important to detect and correct for $\mathrm{MACRO} \sigma^{2} e$ in DI investigations.

Besides MACRO $\sigma^{2} e$, which can produce changes in the DI estimators and changes of the population mean trait size, there is another potential bias that can affect DI estimates. This is another kind of $\mathrm{EH}$ which will be undetectable by the method proposed by Pertoldi et al (2001a) as it is not producing any covariance between the right and left side. If EH does not alter the individual's trait size, but only the DI level then the $\sigma^{2} p$ estimate will be equal to the mean of the individuals DI:

$$
\sigma^{2} p=(\Sigma \mathrm{DI}) / n
$$

Such heterogeneity can, however, be detected by means of an admixture analysis, which will identify the presence of heterogeneity in the distribution of the trait or in the traits' $(r-l)$ distribution (Pertoldi et al, 2005b). With the admixture analysis it will furthermore be possible to detect the presence of dominance interactions within a sample. Therefore, the lack of heterogeneity of a distribution will exclude the possibility that dominance interactions are present.

By removing all the replicates of the experiment where $\mathrm{EH}, \sigma^{2} e, \sigma^{2} g, \mathrm{G} \times \mathrm{E}$, covGE are significantly different from zero, a reliable DI estimate for the two parental lines and for the F1 generation will be obtained. We will also obtain a clear answer on whether heterozygosity (which is assumed to be different from zero in the F1 generation as compared to the two parental populations which are assumed to be totally homozygous) decreases or increases or if it has no effect on the individuals' DI.

We suggest that the method presented here has the potential to increase the value of DI as an assessment tool for the detection of genetic and also environmental stress. In addition, the method can be of great value in connection with other biomarkers and ecotoxicological tests since estimating MACRO $\sigma^{2} e$ and removing samples where a significant MACRO $\sigma^{2} e$ is observed can increase the reproducibility of the standard tests. Whatever the results from the suggested procedure, measures of DI should not be applied uncritically or without an understanding of the nature of the suspected stressors and their effects on fitness (and perhaps a better understanding of developmental mechanisms). Others have also advised caution in the study of DI, often on methodological grounds (Markow, 1995; Palmer, 1996).

DI has been interpreted as a bet-hedging strategy in an unpredictable environment (Simmons and Johnston, 1997). Increased DI with increased stress could also be considered in an evolutionary context as a mechanism of defense for populations in fluctuating environments. The apparent lack of response to selection observed for traits in some sexually reproducing species could be explained by a high degree of DI and/or phenotypic plasticity that would obscure selective differences among genotypes (Sultan, 1996). Developmental instability and phenotypic plasticity can mask genetic differences among individuals. In such a way, the population protects itself against fast changes of genetic structure produced by environmental changes. That would imply a smaller evolutionary potential. However, when the magnitude of genetic variation is insufficient to create a diversity of phenotypes that can be exposed to selection, DI, by producing stochastic variation, will enrich the evolutionary potential of a population. Stochastic fluctuations will contribute to the development of extreme phenotypes by exposing them to selection, thus affecting frequencies of the corresponding genotypes (and contribute to lower phenotypic variation).

\section{Conclusion}

Several meta-analyses have been performed to test for associations between DI and heterozygosity and a negative relationship between DI and heterozygosity has often been suggested (Britten, 1996; Vøllestad et al, 1999). A large number of studies have, however, failed to support the existence of a positive relationship between 
the level of genetic stress and DI, and furthermore many such results ('negative' results) may not be represented in the literature. Hence, the debate about the association between $\sigma^{2} g$ and DI is still open, and because of the problems associated with publication bias, further metaanalysis will not add much to this problem.

\section{Acknowledgements}

The work was supported by grants from the Danish Natural Sciences Research Council. We are grateful to Stuart Barker and Kasper Breuker for helpful comments on the manuscript.

\section{References}

Andersen DH, Pertoldi C, Scali V, Loeschcke V (2002). Intraspecific hybridisation, developmental stability and fitness in Drosophila mercatorum. Evol Ecol Res 4: 603-621.

Britten HB (1996). Meta-analyses of the association between multilocus heterozygosity and fitness. Evolution 50: 21582164

Burke JM, Arnold ML (2001). Genetics and the fitness of hybrids. Annu Rev Genet 35: 31-52.

Clarke GM (1997). The genetic basis of developmental stability. III. Haplo-diploidy: are males more unstable than females? Evolution 51: 2021-2028.

Clarke GM (1998a). The genetic basis of developmental stability. IV. Individual and population asymmetry parameters. Heredity 80: 553-561.

Clarke GM (1998b). The genetic basis of developmental stability. V. Inter- and intra-individual character variation. Heredity $\mathbf{8 0}$ : 562-567.

Clarke GM (2003). Developmental stability-Fitness relationships in animals: some theoretical considerations. In: Polak M (ed) Developmental instability: Causes and consequences. Oxford University Press: New York. pp 187-195.

Crespi BJ, Brett AV (1997). Fluctuating asymmetry in vestigial and functional traits of a haplodiploid insect. Heredity $\mathbf{7 9}$ 624-630.

Crnokrak P, Roff DA (1999). Inbreeding depression in the wild. Heredity 83: 260-270.

Deng HW (1997). Increase in developmental instability upon inbreeding in Daphnia. Heredity 78: 182-189.

Edmands S (1999). Heterosis and outbreeding depression in interpopulation crosses spanning a wide range of divergence. Evolution 53: 1757-1768.

Falconer DS, Mackay (1996). Introduction to Quantitative Genetics, 4th edn. Longman Inc.: New York. pp 464.

Ferguson MM, Danzmann RG, Allendorf FW (1987). Developmental stability of hybrids between two taxa of salmonid fishes with moderate structural gene divergence. Can J Zool 66: 1389-1395.

Frankham R (2005). Genetics and extinction. Biol Conserv 126: 131-140.

Gavrilets S, Hastings A (1994). A quantitative-genetic model for selection on developmental noise. Evolution 48: 1478-1486.

Gilligan DM, Woodworth LM, Montgomery ME, Nurthern RK, Briscoe DA, Frankham R (2000). Can fluctuating asymmetry be used to detect inbreeding and loss of genetic diversity in endangered populations? Anim Cons 3: 97-104.

Hedrick PW, Miller PS (1992). Conservation genetics: techniques and fundamentals. Ecol Applic 2: 30-46.

Keller L, Waller DM (2002). Inbreeding effects in wild populations. Trends Ecol Evol 17: 230-241.

Kristensen TN, Pertoldi C, Pedersen LD, Andersen DH, Bach LA, Loeschcke V (2004). The increase of fluctuating asymmetry in a monoclonal strain of collembolans after chemical exposure - discussing a new method for estimating the environmental variance. Ecol Indicat 4: 73-81.
Kristensen TN, Sørensen AC, Sorensen D, Pedersen KS, Sørensen JG, Loeschcke V (2005). A test of quantitative genetic theory using Drosophila - effects of inbreeding and rate of inbreeding on heritabilities and variance components. J Evol Biol 18: 763-770.

Kruuk LEB, Slate J, Pemberton JM, Clutton-Brock TH (2003). Fluctuating asymmetry in a secondary sexual trait: no associations with individual fitness, environmental stress or inbreeding, and no heritability. I Evol Biol 16: 101-113.

Lamb T, Novak JM, Mahoney DL (1990). Morphological asymmetry and interspecific hybridization: a case study using hylid frogs. J Evol Biol 3: 295-309.

Leary RF, Allendorf FW, Knudsen KL (1983). Developmental stability and enzyme heterozygosity in rainbow trout. Nature 301: 71-72.

Lens L, Van Dongen S, Galbusera P, Schenck T, Matthysen E, Van de Casteele T (2000). Developmental instability and inbreeding in natural bird populations exposed to different levels of habitat disturbance. J Evol Biol 13: 889-896.

Lens L, Van Dongen S, Kark S, Matthyssen E (2002a). Fluctuating asymmetry as an indicator of fitness: can we bridge the gap between studies? Biol Rev 77: 27-38.

Lens L, Van Dongen S, Matthyssen E (2002b). Fluctuating asymmetry as an early warning system in the critically endangered Taita thrush. Cons Biol 16: 479-487.

Lerner IM (1954). Genetic Homeostasis. Oliver and Boyd: Edinburgh, Scotland.

Leung B, Knopper L, Mineau P (2003). A critical assessment of the utility of fluctuating asymmetry as a biomarker of anthropogenic stress. In: Polak M (ed) Developmental Instability: Causes and Consequences. Oxford University Press: New York. pp 415-426.

Livshits G, Kobyliansky E (1985). Lerner's concept of developmental homeostasis and the problem of heterozygosity level in natural populations. Heredity 55: 341-353.

Livshits G, Smouse PE (1993). Relationship between fluctuating asymmetry, morphological modality and heterozygosity in an elderly Israeli population. Genetica 89: 155-166.

Lynch M (1991). The genetic interpretation of inbreeding depression and outbreeding depression. Evolution 45: 622-629.

Markow TA (1995). Evolutionary ecology and developmental instability. Ann Rev Ent 40: 105-120.

Miller PS, Hedrick PW (1993). Inbreeding and fitness in captive populations: lessons from Drosophila. Zoo Biol 12: 333-351.

Palmer AR (1996). Waltzing with asymmetry. Bioscience 46: 518-532.

Palmer RA (1994). Fluctuating asymmetry analyses: a primer. In: Markow TA (ed) Developmental Instability: its Origin and Evolutionary Implications. Kluwer Academic: Netherlands. pp 335-364.

Parsons PA (1992). Fluctuating asymmetry: a biological monitor of environmental and genomic stress. Heredity 68: 361-364.

Pertoldi C, Andersen DH, Kristensen TN, Loeschcke V (2003). Consequences of a reduction in genetic variance on developmental instability estimators. Evol Ecol Res 5: 893-902.

Pertoldi C, Andersen DH, Røgilds A, Loeschcke V (2005a). Heat induced maternal effects in Drosophila mercatorum and its evolutionary consequences. Evol Ecol Res 7: 203-217.

Pertoldi C, García-Perea R, Godoy A, Delibes M, Loeschcke V (2005b). Morphological consequences of range fragmentation and population decline on the endangered Iberian lynx (Lynx pardinus). J Zool (in press).

Pertoldi C, Kristensen TN, Loeschcke V (2001a). A new method for estimating environmental variability for parthenogenetic organisms, and the use of fluctuating asymmetry as an indicator of developmental stability. J Theor Biol 4: 407-410.

Pertoldi C, Loeschcke V, Scali V (2001b). Developmental stability in sexually reproducing and parthenogenetic populations of Bacillus rossius rossius and Bacillus rossius redtenbacheri. Evol Ecol Res 4: 449-463. 
Rao GY, Andersson S, Widén B (2002). Developmental stability in Brassica cretica: the effect of crossing distance on fluctuating asymmetry in cotyledon morphology. Heredity 88: 197-202.

Roff DA (1998). Effects of inbreeding on morphological and life history traits of the sand cricket, Gryllus firmus. Heredity 81: 28-37.

Rutherford SL (2003). Between genotype and phenotype: protein chaperones and evolvability. Nat Rev Genet 4: 263-274.

Rutherford SL, Lindquist S (1998). Hsp90 acts as a capacitor for morphological evolution. Nature 396: 336-342.

Simmons AM, Johnston MO (1997). Developmental stability as a bet-hedging strategy. Oikos 80: 401-406.

Spielman D, Brook BW, Frankham R (2004). Most species are not driven to extinction before genetic factors impact them. Proc Nat Acad Sci USA 101: 15261-15264.
Sultan SE (1996). Evolutionary implications of phenotypic plasticity in plants. I Evol Biol 21: 127-178.

Taylor DS (2001). Physical variability and fluctuating asymmetry in heterozygous and homozygous populations of Rivulus marmoratus. Can J Zool 79: 766-778.

Vøllestad LA, Hindar K, Møller AP (1999). A meta-analysis of fluctuating asymmetry in relation to heterozygosity. Heredity 83: 206-218.

Waser NM, Williams CF (2001). Inbreeding and outbreeding. In: Fox CW, Roff DA, Fairbairn, DJ (eds) Evolutionary Ecology, Concepts and Case Studies. Oxford University Press: Oxford, UK. pp 84-96.

Whitlock M (1998). The repeatability of fluctuating asymmetry: a revision and extension. $P$ Roy Soc Lond B 265: 1428-1430.

Whitlock MC, Fowler K (1999). The changes in genetic and environmental variance with inbreeding in Drosophila melanogaster. Genetics 152: 345-353. 